\title{
Design of an Antipodal Vivaldi Antenna Focusing on Constructional Aspects
}

\author{
David S. Cabral $^{1}\left(\mathbb{D}\right.$, Leandro Manera ${ }^{2}$, Leonardo B. Zoccal ${ }^{3}\left(\mathbb{D}\right.$, Daniel B. Ferreira ${ }^{4}$, \\ Francesco Prudenzano ${ }^{5}$ (D) \\ ${ }^{1}$ Telescope Services Ab, Scheelevägen 16, 223 63, Lund, Sweden, david.cabral@telescopeservices.se \\ ${ }^{2}$ Semiconductors, Instrumentation and Photonics Department - DSIF, State University of Campinas, Albert \\ Einstein Avenue, 400, FEEC, Campinas, Brazil, manera@fee.unicamp.br \\ ${ }^{3}$ Institute of Systems Engineering and Information Technology - IESTI, Federal University of Itajuba, 1303 BPS \\ Avenue, P.O. Box 50, IESTI-UNIFEI, Itajuba, Brazil, lbzoccal@unifei.edu.br \\ ${ }^{4}$ Department of Microwaves and Optoelectronics, Aeronautics Institute of Technology, Pca Mal Eduardo \\ Gomes, 50, Sao Jose dos Campos, Brazil, danielbf@ita.br \\ ${ }^{5}$ Department of Electrical and Information Engineering - DEI, Polytechnic University of Bari, Via Orabona, 4, \\ Bari, Italy, francesco.prudenzano@poliba.it
}

\begin{abstract}
This paper presents an Antipodal Vivaldi Antenna (AVA) design, focusing on its constructional aspects. The main features analysed are the connector attachment structure and the introduction of a polytetrafluoroethylene (PTFE) part that supports the antenna laminate. Issues related to dielectric penetration by milling tools are also addressed. The proposed AVA was manufactured through a low-cost prototyping process and tested, achieving an operational bandwidth from 5 to $18 \mathrm{GHz}$ for a reflection coefficient less than $-10 \mathrm{~dB}$ and an average gain of 6.23 dBi. The prototype meets all design requirements, which shows the viability of the developed radiator.
\end{abstract}

Index Terms - AVA design, Antipodal Vivaldi, Full-wave simulations, UWB Antenna

\section{INTRODUCTION}

Ultra-wideband (UWB) antennas have been used in various applications over the last years [1][4]. Military applications, such as phased array radars, direction finding systems, and see-through-wall imaging radars, benefit UWB antennas [5], [6]. Among the most popular UWB radiators is the tapered slot antenna (TSA) with its various profiles, including the exponential taper profile, first proposed in [7], and well-known as Vivaldi antenna [8]. One simple and effective way to feed Vivaldi antennas is through the antipodal feed technique since it keeps the antenna bandwidth broad and its input impedance low [8], [9]. This so-called Antipodal Vivaldi Antenna (AVA) is an attractive radiator mainly due to its low profile, low cost, low weight, and satisfactory radiation characteristics. However, there are some gaps in antenna literature concerning AVAs' design and manufacture, especially when considering their constructional aspects for high-performance applications [10]-[12].

The installation of antennas and antenna arrays on mobile military platforms, e.g., ships, drones, and aircraft, poses a challenge due to hard-mechanical vibration and thermal conditions [13]-[15]. Moreover, it is not always possible to modify the platform structure to install an antenna or antenna arrays in a more appropriate position with lower vibration levels and temperature variations because it can drastically impair the platform aerodynamics [15]. On the other hand, structural elements added, 
such as plastic supports and metal clamps, enhance the antenna's mechanical robustness; however can degrade its electrical performance, particularly at higher operating frequencies [5].

Many works on specialised literature present different design and layout techniques focusing on exponential flares radiators and feeder structure adjustments to improve AVAs' electrical performance. In [16], a tapered slot edge (TSL) structure was proposed to extend the frequency band's low end and improve the impedance matching and radiation pattern. Furthermore, positioned after the AVA's radiator, lenses using different dielectric material [17], [18] or made directly on the antenna substrate [19], [20] can result in higher directivity. However, the extensive literature is insufficient in providing a quantitative analysis of the final AVA performance when considering a parasitic structure in contact with its surface (e.g., mechanical support) [21]. Additionally, gaps are verified in the study of the AVA performance effects when considering the inherent prototyping errors caused by the manufacturing process through a commercial prototyping drill machine. To the best of our knowledge, no published works deal with this issue for AVAs, unlike microstrip antennas. Some papers discuss possible problems when a prototyping drill machine is employed in the manufacturing phase [22].

In this work, a compact and easy-manufactured AVA is designed with the aid of full-wave simulations. The synthesised antenna operates from $4.5 \mathrm{GHz}$ to $18 \mathrm{GHz}$ showing a reflection coefficient lower than $-10 \mathrm{~dB}$. Mechanical support made of polytetrafluoroethylene (PTFE) is further included in the antenna model, and an aluminium clamp guarantees the antenna's grounding even under high vibration conditions. The influence of these elements on AVA electrical performance is also studied. Another issue addressed in this paper is the tool penetration's effect when the AVA is fabricated using a milling machine. Finally, a prototype, including the mechanical support in PTFE, was fabricated and tested in a near field anechoic chamber. The simulated and measured results are compared to validate the analyses presented in the text.

The rest of this paper is organised as follows. Section II addresses the design equations and the resulting dimensions for the AVA, providing all details to formulate the AVA model. Applied connectors, ground structure and substrate milling depth analyses are shown in Section III. Next, the 3D modelling results of the electrical influence of PTFE mechanical structures coupled to the designed AVA is presented. The resulting E-plane and H-plane patterns and the complete structure's gain are also described in this section. Finally, some conclusions are drawn in Section IV.

\section{Antipodal Vivaldi Antenna Design}

The tapered slot Vivaldi antenna is a broadband end-fire radiator, whose original design was introduced by Gibson in 1979, [8], and is composed of two parts: 1) a thin exponentially tapered metallic profile etched on one side of a laminate, and 2) a matching circuit that is printed on the other side. In turn, the AVAs differ from the previous antenna in the way their radiating structure is printed on both sides of the dielectric substrate [23], [24], as visualised in Fig. 1. The symmetrical tapers that show up on the top and bottom sides represent an exponential profile defined by the following equation [25]

$$
y=C_{1} \mathrm{e}^{R x}+C_{2}
$$

where

$$
C_{1}=\left(y_{2}-y_{1}\right) /\left(\mathrm{e}^{R x_{1}}-\mathrm{e}^{R x_{2}}\right)
$$

Brazilian Microwave and Optoelectronics Society-SBMO

Brazilian Society of Electromagnetism-SBMag received 26 Mar 2021; for review 8 Apr 2021; accepted 11 Aug 2021 (C) 2021 SBMO/SBMag (cc) BY

ISSN 2179-1074 


$$
C_{2}=\left(y_{1} \mathrm{e}^{R x_{2}}-y_{2} \mathrm{e}^{R x_{1}}\right) /\left(\mathrm{e}^{R x_{2}}-\mathrm{e}^{R x_{1}}\right)
$$

and $R$ is an expansion factor whose value establishes a compromise between optimal gain and reflection coefficient; it is usually in the range of 0.25 to $0.45 . P_{1}\left(x_{1}, y_{1}\right)$ and $P_{2}\left(x_{2}, y_{2}\right)$ are the exponential taper's first and last points, respectively, as depicted in Fig. 1. It should be noted that the coordinate system origin is centred at the feed line end and coincides with the cutting $A-A$ plane centre. Thus, these points can be expressed as $P_{1}\left(W_{f} / 2,0\right)$ and $P_{2}\left(W_{b}-W / 2, L\right) . W_{b}$ is adjusted to determine the $P_{2}$ position and consequently impacts the lower operating frequency of the antenna. Additionally, $L_{b}$ and $R_{b}$ parameters are set to guarantee the desired 50- $\Omega$ input impedance and provide a radiation pattern with good compliance.

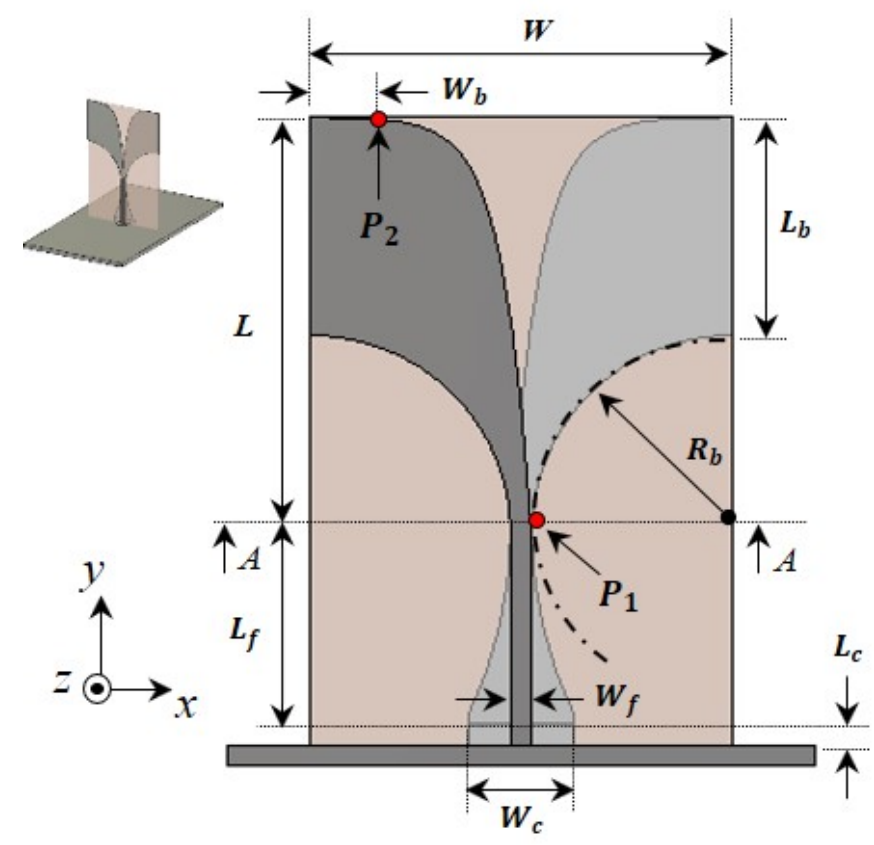

Fig. 1. AVA geometry with an aluminium reflector.

For an AVA to behave as an effective surface wave antenna, the following condition involving the effective substrate thickness $t_{\text {eff }}$ must hold [26]:

$$
0.005<t_{e f f} / \lambda_{0}=\left(\sqrt{\varepsilon_{r}}-1\right) h / \lambda_{0}<0.03
$$

in which $\varepsilon_{r}$ and $h$ are the substrate relative permittivity and thickness, respectively, and $\lambda_{0}$ is the free-space wavelength at the operating range's centre frequency. For $t_{e f f} / \lambda_{0} \geq 0.03$, the high contrast between the antenna dielectric and free space is too drastic, resulting in large reflections back into the antenna [27].

An initial estimate for the antenna length $(L)$ can be obtained by [27]:

$$
L=c / f_{\min } \sqrt{2 /\left(\varepsilon_{r}+1\right)}
$$

where $f_{\min }$ is the lowest operating frequency, and $c$ is the free-space speed of light. In this paper's design, the antenna width $(W)$ was made equal to $L$. In Fig. 1, a transition line was placed between the feed connector and the $A-A$ plane to convert the unbalanced feed structure to a balanced parallel 
line. In this design, the top conductor width $W_{f}$ does not change along its length, while the bottom conductor edges follow an exponential profile with an expansion factor of 0.01 , and its end widths are $W_{f}$ and $W_{c}=5 W_{f}$. $L_{f}$ 's transition line length was fixed at $0.2 \lambda_{f_{\min }}\left(\lambda_{f_{\min }}\right.$ is the substrate's wavelength at the lowest operating frequency), which guarantees a proper impedance matching and a controlled beam squint.

The AVA was designed to operate from 6 to $18 \mathrm{GHz}$ since this band is of great interest for military applications [28]. A reflection coefficient magnitude better than $-10 \mathrm{~dB}$ over this band and gain above 7 $\mathrm{dBi}$ were required. As seen from Fig. 1, a custom aluminium reflector $\left(53 \times 100 \times 30 \mathrm{~mm}^{3}\right)$ was included in the antenna model to minimise the back-radiation.

Considering the adopted frequency range and assuming the use of Rogers RT/Duroid 5870 microwave laminate (relative permittivity of 2.33 , loss tangent of 0.0012 , and thickness of $0.787 \mathrm{~mm}$ ), the antenna dimensions were initially evaluated with the aid of (1) to (5). The obtained dimensions are depicted in Table I, and the effective substrate thickness $t_{e f f}$ is equal to $0.014 \lambda_{0}$, thus satisfying (4).

Next, the initial dimensions and the expansion factor $R$ were optimised using a full-wave analysis tool based on the finite element method (FEM) called Dassault CST Studio. The optimisation process started at the AVA and later at the feed line, which can be viewed at the top and bottom of the $A-A$ plane in Fig.1, respectively. In the AVA optimisation process, the two tapers were driven by using a discrete port in CST. Starting from the calculated value of $W$, the values of $W_{b}$ and $R_{b}$ were initially chosen as $0.25 \mathrm{~W}$ and $0.45 \mathrm{~W}$, respectively. $R$ was parameterised and varied from 0.1 to $0.5 \mathrm{~mm}$ with a $0.05 \mathrm{~mm}$ step size. Similarly, $W_{b}$ and $R_{b}$ were parameterised and varied $\pm 20 \%$ of their initial values with $0.05 \mathrm{~mm}$ step size. $W$ and $L$ have been adjusted to be integers and have the same value. The parameter $W_{f}$ was, in turn, varied $\pm 15 \%$ of its initial value with a step of $0.05 \mathrm{~mm}$.

To guide the parameters' optimisation, the distance between the power reflection coefficient curve and the threshold of $-10 \mathrm{~dB}$, in a least-squares sense, was maximised, i.e.,

$$
S_{\text {opt }}=\arg \max _{S}\left\{\sum_{k=1}^{K}\left(\left|\Gamma\left(f_{k}\right)\right|^{2}-0.1\right)^{2}\right\}
$$

in which $S_{\text {opt }}$ denotes the set of optimum values of the antenna parameters, i.e., $S=\left\{W, W_{b}, R_{b}, W_{f}, R\right\}$, $\Gamma$ is the reflection coefficient, and the frequencies $f_{k}(k=1, \ldots, K)$ are uniformly sampled over the frequency range of 6 to $18 \mathrm{GHz}$. The optimised parameters are also presented in Table I. It is worth noting that the antenna gain was less sensitive to the parameters variation than the reflection coefficient. Following, the feed line was included in the simulation model with the parameters defined above and the length $L_{f}$ was slightly increased to control the main lobe squint.

TABLE I. ESTIMATED AND OPTIMISED AVA DESIGN PARAMETERS

\begin{tabular}{ccc}
\hline Parameter & Initial value & Optimised values \\
\hline$W=L$ & $38.52 \mathrm{~mm}$ & $40.00 \mathrm{~mm}$ \\
$W_{b}$ & $3.85 \mathrm{~mm}$ & $4.00 \mathrm{~mm}$ \\
$W_{f}$ & $2.12 \mathrm{~mm}$ & $2.00 \mathrm{~mm}$ \\
$W_{c}$ & $10.60 \mathrm{~mm}$ & $10.00 \mathrm{~mm}$ \\
$L_{b}$ & $19.26 \mathrm{~mm}$ & $21.00 \mathrm{~mm}$ \\
$R$ & 0.30 & 0.36 \\
$L_{f}$ & $19.26 \mathrm{~mm}$ & $20.50 \mathrm{~mm}$ \\
$L_{c}$ & $3.00 \mathrm{~mm}$ & $3.00 \mathrm{~mm}$ \\
\hline
\end{tabular}

Brazilian Microwave and Optoelectronics Society-SBMO Brazilian Society of Electromagnetism-SBMag received 26 Mar 2021; for review 8 Apr 2021; accepted 11 Aug 2021 (C) 2021 SBMO/SBMag (cc) BY

ISSN 2179-1074 
Fig. 2 shows the simulated reflection coefficient magnitude and gain for the optimised AVA model. The proposed antenna presents excellent UWB performance. It exhibits a reflection coefficient lower than $-10 \mathrm{~dB}$ from $4.96 \mathrm{GHz}$ to $20 \mathrm{GHz}$ and an average gain of $9.0 \mathrm{dBi}$ in the operational range of 6 $\mathrm{GHz}$ to $20 \mathrm{GHz}$. Consequently, the results exceed the proposed performance requirements. Furthermore, the simulated AVA radiation efficiency is above $90 \%$ in the operational range of $6 \mathrm{GHz}$ to $20 \mathrm{GHz}$. Above $10 \mathrm{GHz}$, the maximum and minimum simulated gain were 9.90 and $8.28 \mathrm{dBi}$, respectively.

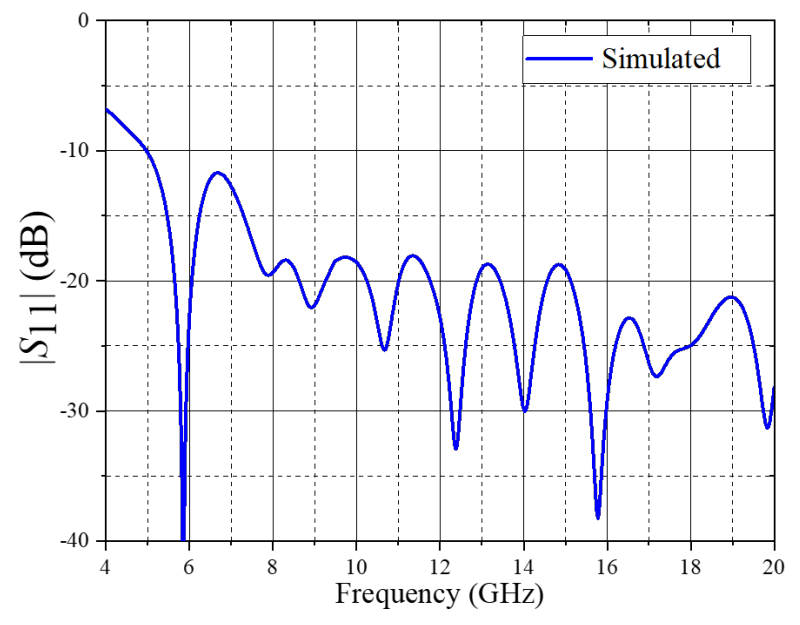

(a)

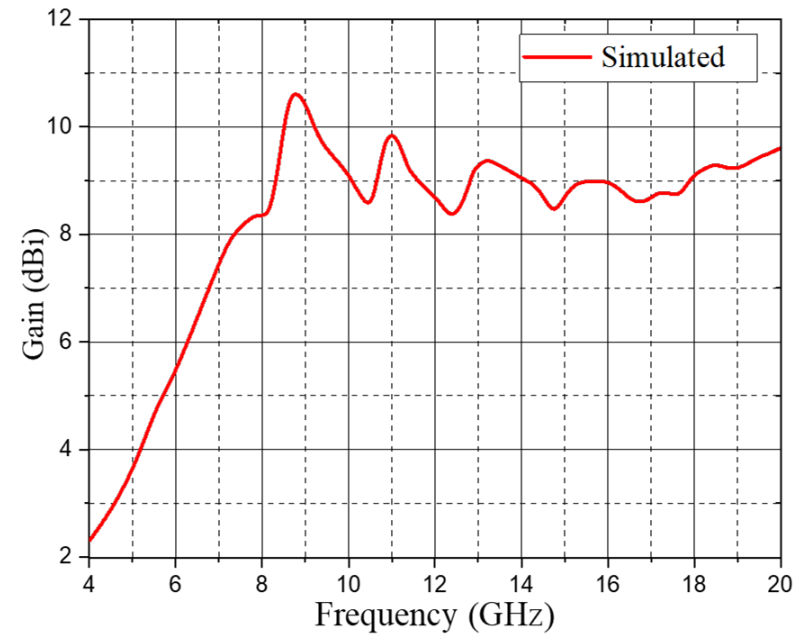

(b)

Fig. 2. (a) Reflection coefficient magnitude and (b) gain for the optimised AVA model.

\section{Constructional Aspects Analysis}

\section{A. Ground Structures}

As previously mentioned, the AVA developed in this work must withstand high vibration levels. Therefore, to ensure adequate grounding and improve mechanical robustness, two different ground solutions were proposed. The impacts of these solutions on the electrical antenna performance are presented in this section.

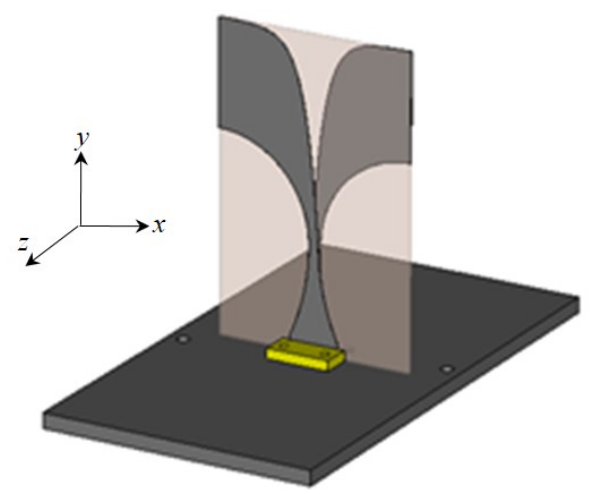

(a)

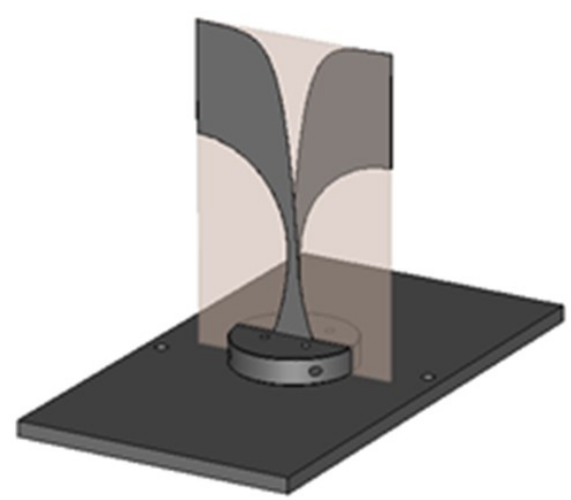

(b)

Fig. 3. 3D AVA models with mechanical supports. (a) AVA ground mechanical solution 1 (RMP), (b) AVA ground mechanical solution 2 (CMP).

Brazilian Microwave and Optoelectronics Society-SBMO Brazilian Society of Electromagnetism-SBMag received 26 Mar 2021; for review 8 Apr 2021; accepted 11 Aug 2021 (C) 2021 SBMO/SBMag (cc) BY 
A $50-\Omega$ SMA connector is used to feed the AVA, and its centre conductor is welded parallel to the laminate. Fig. 3 shows the models of both devised ground structures. The rectangular mechanical ground profile (RMP), which can be made of brass, presents itself as a more straightforward solution than the circular mechanical ground profile (CMP). However, CMP provides a more robust mechanical alternative and consequently less susceptible to vibration effects. CMP is not welded on the AVA ground plane as an added advantage, thus avoiding additional thermal stress to the dielectric substrate. CMP can be fabricated with aluminium as the antenna reflector.

Fig. 4 (a) shows the RMP mechanical structure. It is fixed to the reflector using two screws, and one of its sides faces is welded to the antenna's ground plane. $W_{1}$ and $L_{1}$ are the width and the length, respectively. The fixing roles $d_{1}$ and the brass structured thickness $h_{1}$ are presented. Next, Fig. 4 (b) shows CMP mechanical structure where two aluminium half-cylinders are connected with two screws. $d_{2}$ and $W_{1}$ are the external structure diameter and the gap to place the dielectric substrate, respectively. The fixing connector roles $d_{1}$ and the structure thickness $h_{1}$ are also shown in the figure. All mentioned parameters values are described in Table II.

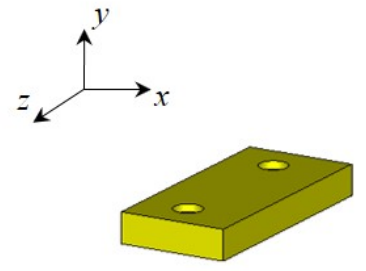

Perspective view

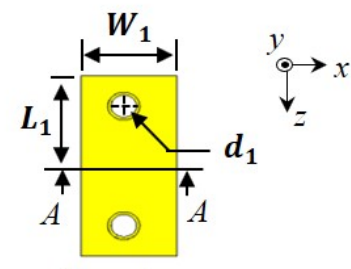

Top view

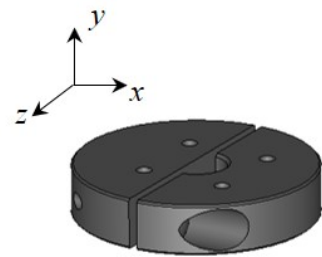

Perspective view

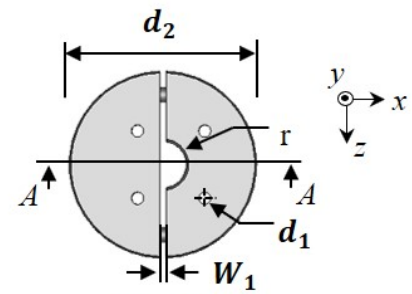

Top view

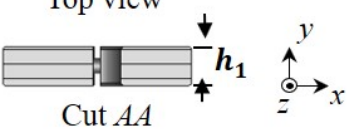

(b)

(a)

Fig. 4. (a) RMP ground mechanical geometry, (b) CMP ground mechanical geometry.

TABLE II. CMP AND RMP MECHANICAL GROUND SOLUTION DIMENSIONS

\begin{tabular}{clllllc}
\hline Parameter & $W_{1}$ & $h$ & $d_{1}$ & $d_{2}$ & $r$ & $L_{1}$ \\
\hline CMP & $0.79 \mathrm{~mm}$ & $5.00 \mathrm{~mm}$ & $1.60 \mathrm{~mm}$ & $23.60 \mathrm{~mm}$ & $8.62 \mathrm{~mm}$ & - \\
RMP & $6.00 \mathrm{~mm}$ & $2.00 \mathrm{~mm}$ & $1.60 \mathrm{~mm}$ & - & - & $6.50 \mathrm{~mm}$ \\
\hline
\end{tabular}

Fig. 5 shows the simulation results for the proposed RMP and CMP ground structures' reflection coefficient magnitude. An excellent impedance matching with $\left|S_{11}\right|<-10 \mathrm{~dB}$ was obtained for frequencies ranging from $4.96 \mathrm{GHz}$ to $25 \mathrm{GHz}$ and $4.75 \mathrm{GHz}$ to $25 \mathrm{GHz}$ to RMP and CMP, respectively. As seen, the RMP solution presents simulated $\left|S_{11}\right|$ results slightly better for frequencies from $8 \mathrm{GHz}$ to approximately $19 \mathrm{GHz}$ than CMP structure. However, considering the operational range and the proposed application, there are no substantial $\left|S_{11}\right|$ improvements. Additionally, $\left|S_{11}\right|<-15 \mathrm{~dB}$ is observed for frequencies from $14 \mathrm{GHz}$ to $25 \mathrm{GHz}$ for both structures. Since no significant changes were observed in the impedance matching considering the operational range, it was decided to proceed with the RMP structure as it has a lower manufacturing cost. 


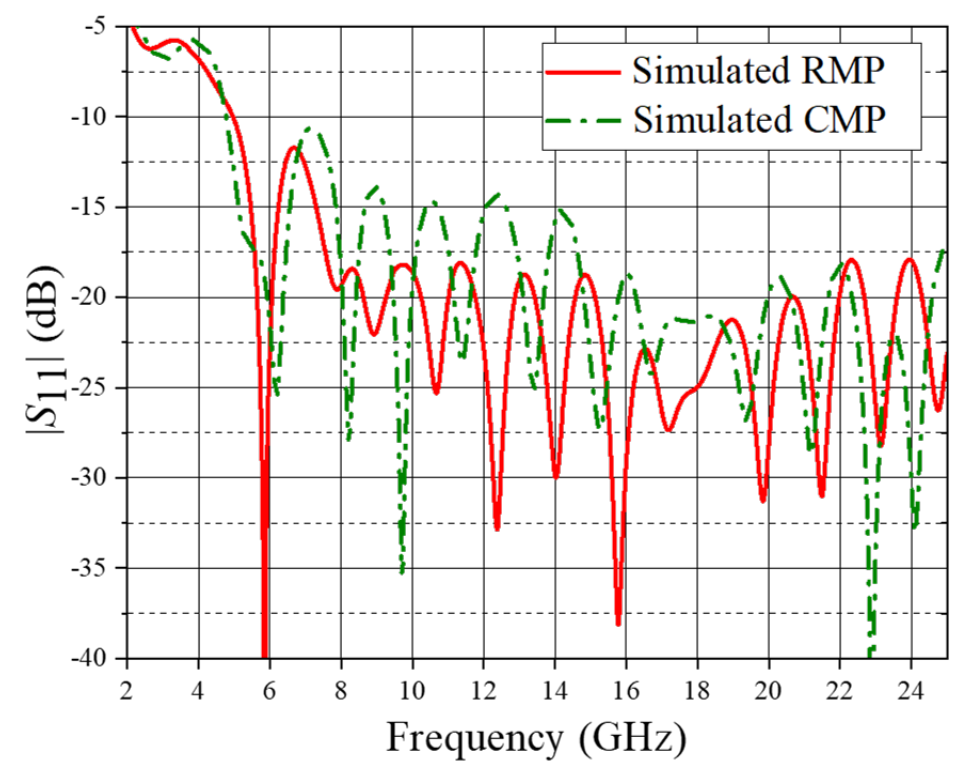

Fig. 5. Simulated reflection coefficients of RMP and CMP.

\section{B. PTFE Support}

As previously stated, this paper proposes a solution to be used in natural environments, where external factors are acting. So, to ensure an appropriate electrical performance, a thin laminate must be used in the design. Consequently, mechanical support is needed to prevent an undesirable beam squint and any damage caused by vibration when the AVA operates in a real application scenario. A PTFE support structure was considered and implemented to the RMP structure, as shown in Fig. 6. This polymer material presents low relative permittivity, $\varepsilon_{r}=2.1$, low loss tangent, high surface resistivity, and good stability at high temperatures [29]. Additionally, it can be easily manufactured in a conventional machining process.

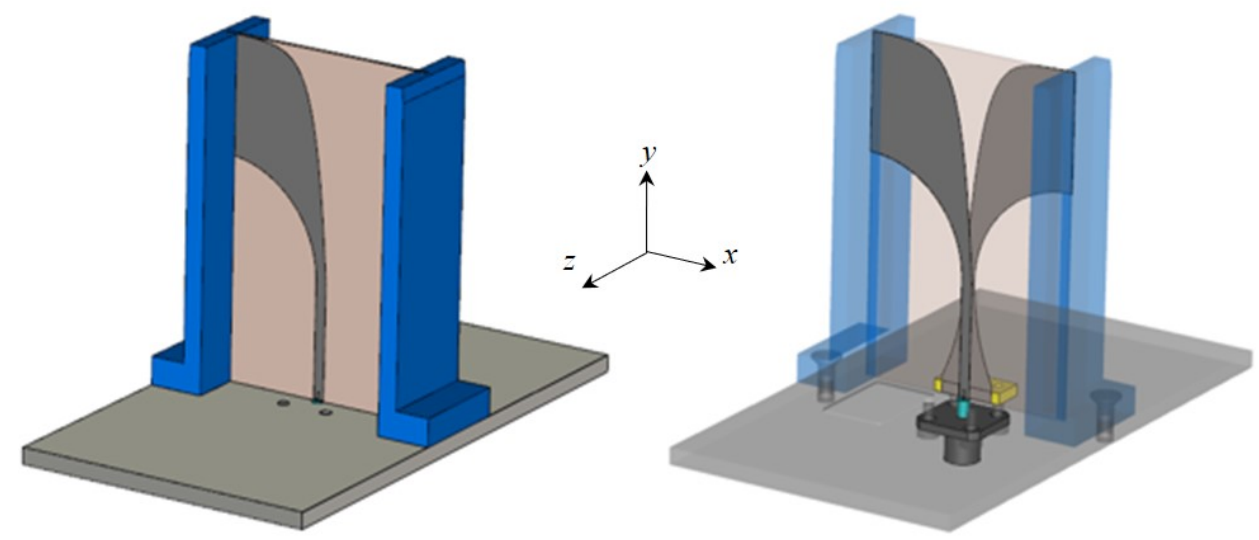

Fig. 6. 3D model of AVA RMP with PTFE support.

To evaluate the support effect on the antenna performance, the radiation patterns (E- \& H-planes) of the AVA co-polarisation $(\mathrm{CoPol})$ and cross polarisation (XPol) components with and without the PTFE mechanical support at different frequencies are presented in Fig. 7. As seen, there are no significant changes between the compared patterns over the frequency range, with the responses at $18 \mathrm{GHz}$ in the H-plane exhibiting the worst degradation. 
Journal of Microwaves, Optoelectronics and Electromagnetic Applications, Vol. 20, No. 4, December 2021 DOI: http://dx.doi.org/10.1590/2179-10742021v20i41270

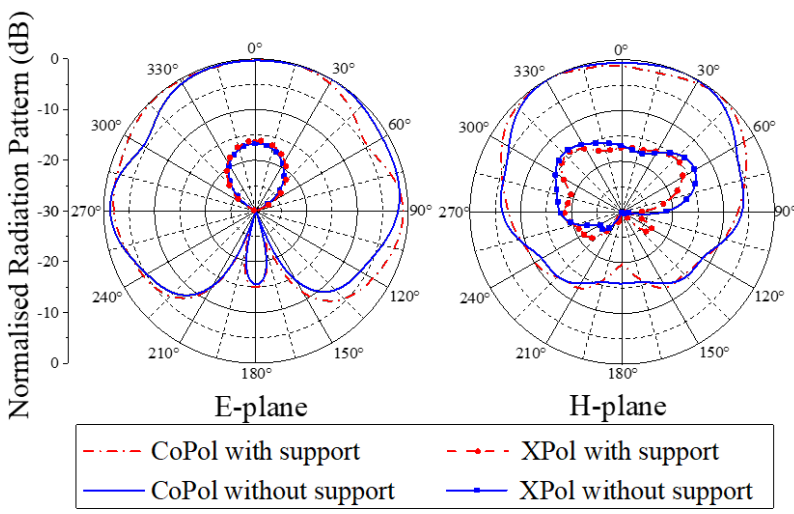

(a)

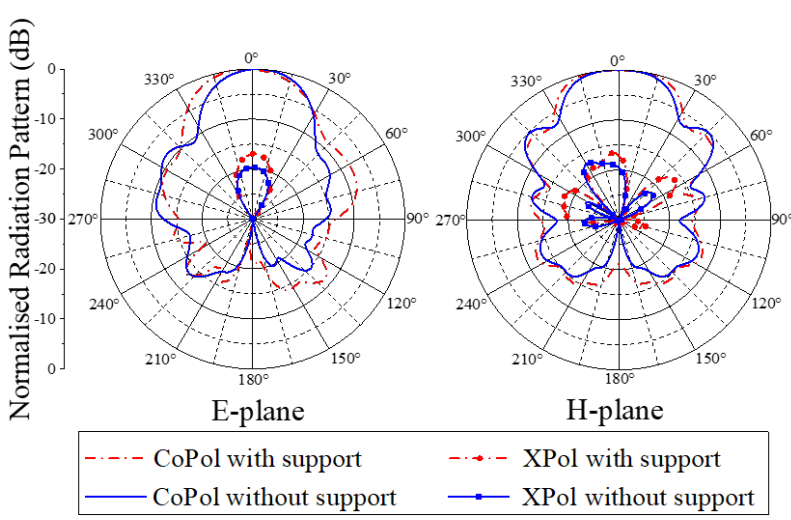

(b)

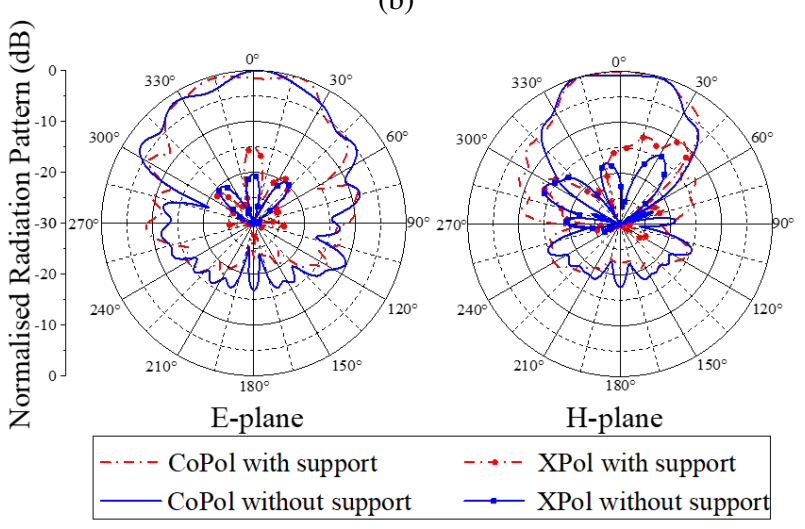

(c)

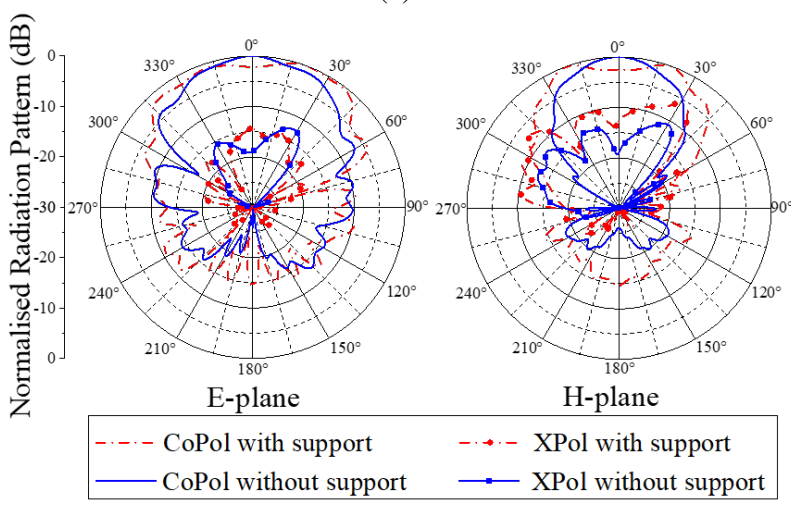

(d)

Fig. 7. Simulated E- and H-plane patterns of the AVA with and without mechanical PTFE structure at (a) 5 GHz, (b) 9 $\mathrm{GHz}$, (c) $14 \mathrm{GHz}$, and (d) $18 \mathrm{GHz}$. 
The AVA was fabricated using a commercial prototyping drill machine. The complete structure was measured in a near field anechoic chamber as visualised in Fig. 8.

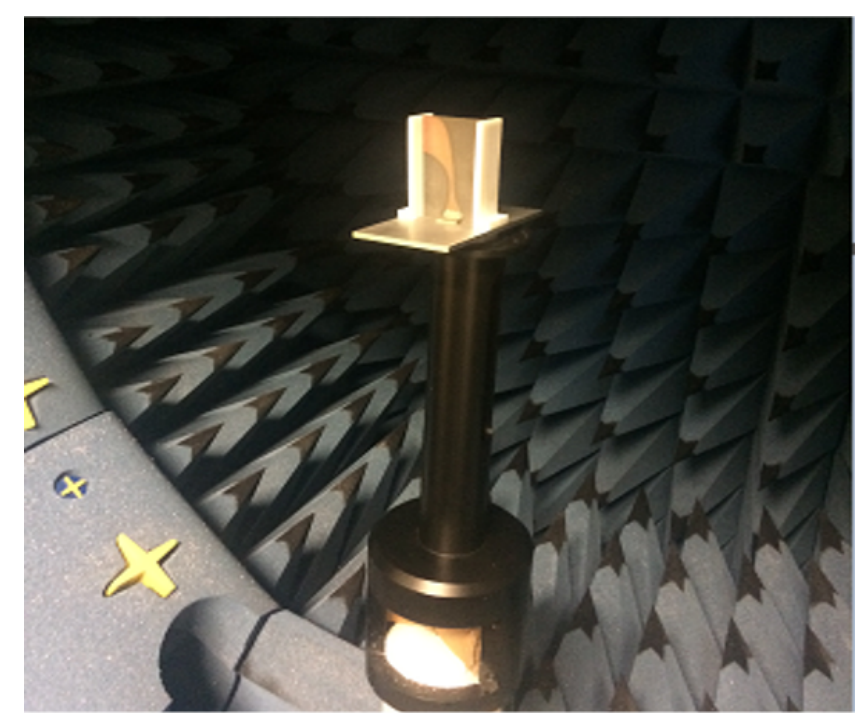

Fig. 8. AVA prototype in the near field anechoic chamber.

Fig. 9 (a) shows the simulation and measured results for the reflection coefficient magnitude. A suitable impedance matching with $\left|S_{11}\right|$ for all measured frequency range was observed. Although the addition of the PTFE support has slightly degraded the antenna response compared to Fig. 2, it remains well-matched over the entire frequency band, presenting a maximum reflection coefficient of $-9.7 \mathrm{~dB}$ at $6.51 \mathrm{GHz}$. Fig. 9 (b) shows a good agreement between simulated and measured gain results of the prototype. The measured peak gain is $10.9 \mathrm{dBi}$ at $8.2 \mathrm{GHz}$, and the radiation structure presents an average gain of $9.0 \mathrm{dBi}$ for the operational range from $6 \mathrm{GHz}$ to $18 \mathrm{GHz}$.

Radiation patterns (E \& H planes) of the AVA co-polarisation (CoPol) with the PTFE mechanical support at different frequencies are presented in Fig. 10. As seen, they are directives in the entire frequency range, and an excellent agreement with the simulated model is observed.

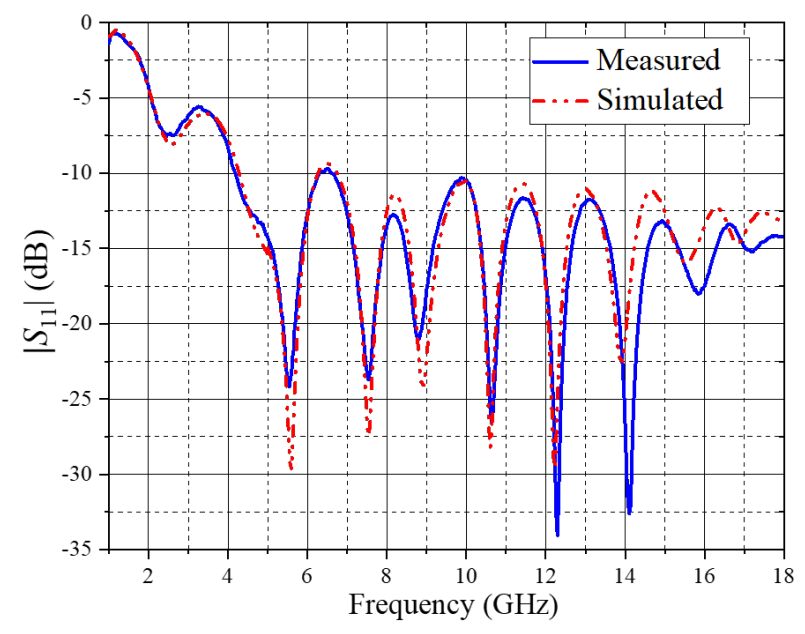

(a)

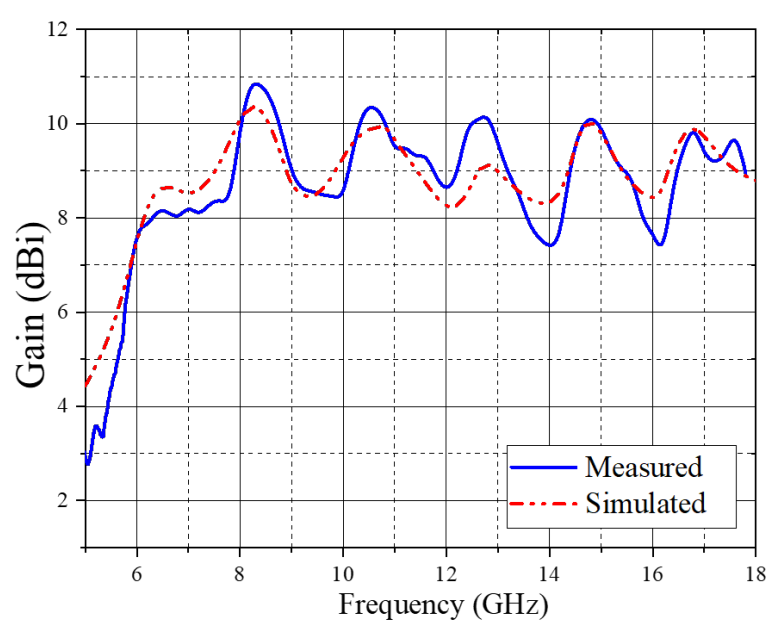

(b)

Fig. 9. (a) Reflection coefficient and (b) gain for the complete AVA structure.

Brazilian Microwave and Optoelectronics Society-SBMO Brazilian Society of Electromagnetism-SBMag received 26 Mar 2021; for review 8 Apr 2021; accepted 11 Aug 2021 (C) 2021 SBMO/SBMag (cc) BY 
Journal of Microwaves, Optoelectronics and Electromagnetic Applications, Vol. 20, No. 4, December 2021 DOI: http://dx.doi.org/10.1590/2179-10742021v20i41270

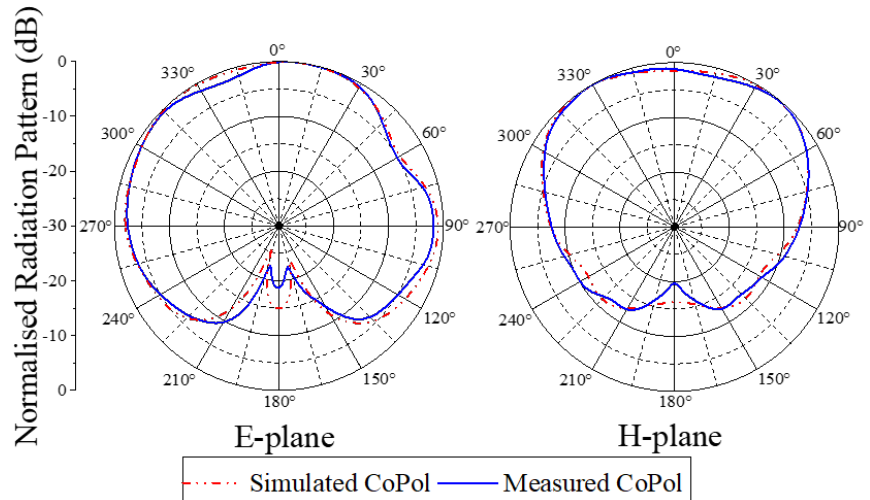

(a)

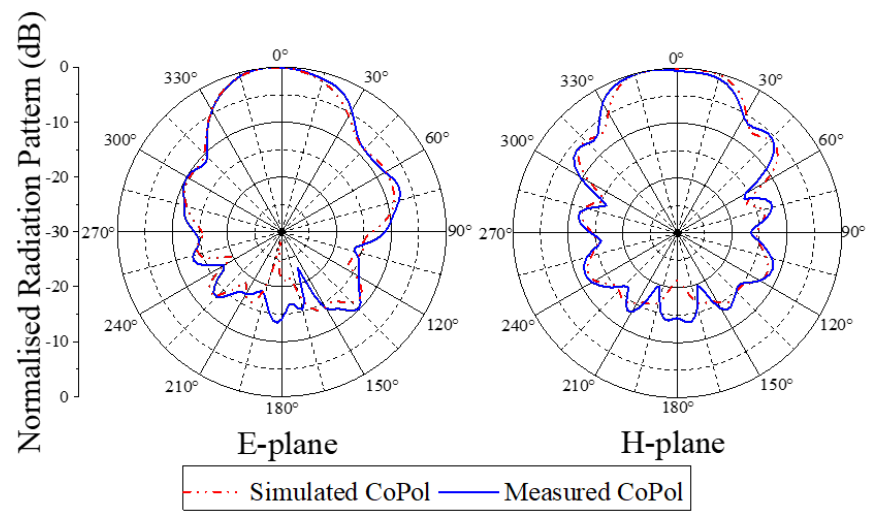

(b)

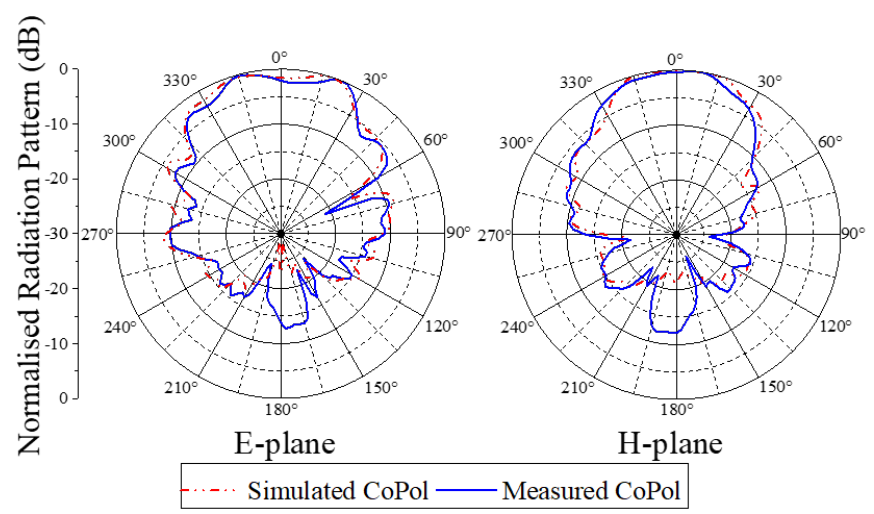

(c)

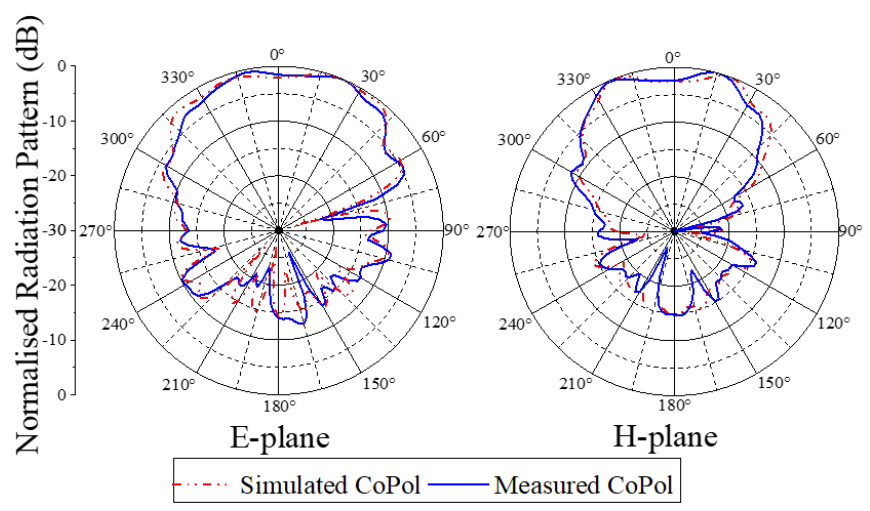

(d)

Fig. 10. E- and H-plane patterns at (a) $5 \mathrm{GHz}$, (b) $9 \mathrm{GHz}$, (c) $14 \mathrm{GHz}$, and (d) $18 \mathrm{GHz}$. 


\section{Substrate Milling Depth}

For this project, a low-cost, fast and easy manufacturing approach was required. The proposed antenna was fabricated from a commercial prototyping drill machine. Fig. 11 depicts a common but often not desired prototyping problem, which is when a drill tool can excessively penetrate the substrate during the prototyping process. This substrate penetration caused by, e.g., poor equipment calibration, results in a linear substrate milling depth. The substrate milling's width and depth vary according to the tool (drill) and inadequate calibration. This occurs along the edge of the drawing to be prototyped. For the analysis carried out, a simulation was performed considering a fixed drill width of $0.381 \mathrm{~mm}$ and a depth varying from 0 to $0.175 \mathrm{~mm}$ with steps of $0.035 \mathrm{~mm}$. Additionally, an extreme case was simulated where a substrate milling depth of $0.750 \mathrm{~mm}$ was applied with the same drill width, representing the removal of $95.29 \%$ of the dielectric.

The simulated reflection coefficient results for drill penetrations are presented in Fig. 12. As can be seen, unlike other topologies, e.g. microstrip antennas [22], the AVA tolerates a removal of dielectric material close to the conductors without significantly disturbing its electrical response. Therefore, such behaviour presents itself as a relevant TSA manufacturing advantage, especially for the proposed AVA in this paper.

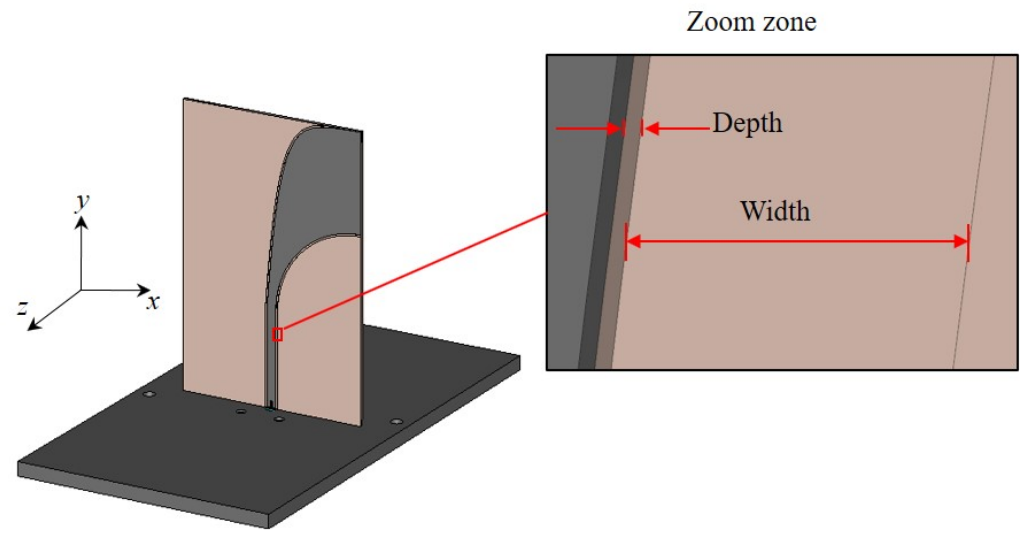

Fig. 11. AVA substrate milling depth geometry.

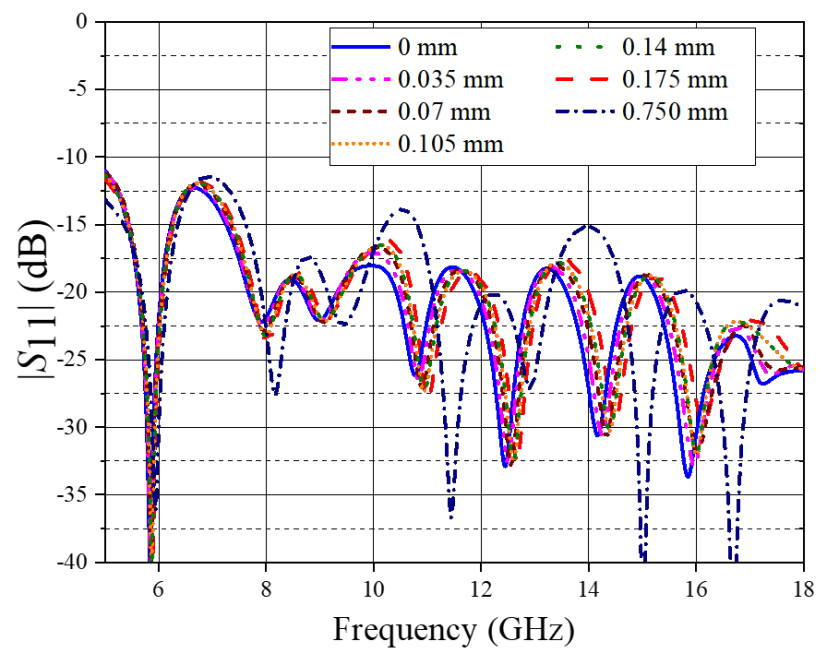

Fig. 12. Simulated reflection coefficients of the antenna with and without substrate milling depth. 


\section{CONCLUSIONS}

A canonical design of an antipodal Vivaldi antenna focused on mechanical grounding and vibration solution aspects has been presented and experimentally validated. The analytic antenna model and the antenna and feed line optimisation approach were discussed. Two different mechanical ground structures were proposed, and they were investigated in this paper. Excellent impedance matching with $\left|S_{11}\right|<$ $-10 \mathrm{~dB}$ over the required operating frequency range from $6 \mathrm{GHz}$ to $18 \mathrm{GHz}$ for both structures was observed. The AVA design presented in this paper showed good compatibility with a fast prototyping machine and revealed itself as a robust solution, even when considered the substrate milling depth problem. Different milling depth penetration and an extreme case were analysed, and the AVA kept a good performance over the operating frequency range. Besides, PTFE mechanical support was proposed as a potential mechanical solution to evaluate the antenna protection against mechanical vibration. The performance results show that even with the PTFE structure, the AVA meets the project requirement with an average gain of $9.0 \mathrm{dBi}$ for the operational range from $6 \mathrm{GHz}$ to $18 \mathrm{GHz}$, and low pattern degradation was observed.

\section{ACKNOWLEDGEMENT}

The authors would like to thank Mr Dario Laneve for his valuable help with the measurements.

\section{REFERENCES}

[1] B. J. Mohammed, A. M. Abbosh, S. Mustafa, and D. Ireland, "Microwave system for head imaging," IEEE Transactions on Instrumentation and Measurement, vol. 63, no. 1, pp. 117-123, 2013.

[2] M. Abbak, M. Akıncı, M. Çayören, and İ. Akduman, "Experimental microwave imaging with a novel corrugated vivaldi antenna," IEEE Transactions on Antennas and Propagation, vol. 65, no. 6, pp. 3302-3307, 2017.

[3] E. W. Reid, L. Ortiz-Balbuena, A. Ghadiri, and K. Moez, "A 324-element vivaldi antenna array for radio astronomy instrumentation," IEEE Transactions on Instrumentation and Measurement, vol. 61, no. 1, pp. 241-250, 2011.

[4] O. Turkmen-Kucuksari, A. Kocakaya, S. Çimen, and G. Çakır, "High constant gain modified antipodal vivaldi antenna incorporated with a phase compensation lens and a frequency selective surface," AEU - International Journal of Electronics and Communications, vol. 113, pp. 152-975, 2020.

[5] Y. Wang, Y. Yang, and A. E. Fathy, "Ultra-wideband vivaldi arrays for see-through-wall imaging radar applications," in 2009 IEEE Antennas and Propagation Society International Symposium, pp. 1-4, 2009.

[6] S. Guruswamy, R. Chinniah, and K. Thangavelu, "A printed compact uwb vivaldi antenna with hemi cylindrical slots and directors for microwave imaging applications," AEU - International Journal of Electronics and Communications, vol. 110, pp. 152-870, 2019.

[7] M. H. H. Agahi, H. Abiri, and F. Mohajeri, "Investigation of a new idea for antipodal vivaldi antenna design," International Journal of Computer and Electrical Engineering, vol. 3, no. 2, p. 277, 2011.

[8] P. Gibson, "The vivaldi aerial," in 1979 9th European Microwave Conference, pp. 101-105, 1979.

[9] A. Bhattacharjee, A. Bhawal, A. Karmakar, A. Saha, and D. Bhattacharya, "Vivaldi antennas: a historical review and current state of art," International Journal of Microwave and Wireless Technologies, p. 1-18, 2020.

[10] M. Moosazadeh and S. Kharkovsky, "Design of ultra-wideband antipodal vivaldi antenna for microwave imaging applications," in 2015 IEEE International Conference on Ubiquitous Wireless Broadband (ICUWB), pp. 1-4, 2015.

[11] S. K. Ghosh and R. K. Badhai, "Design of a novel antipodal vivaldi antenna with further modifications for notch characteristics," in 2017 International Conference on Inventive Systems and Control (ICISC), pp. 1-5, 2017.

[12] Z. Yang, H. Jingjian, W. Weiwei, and Y. Naichang, "An antipodal vivaldi antenna with band-notched characteristics for ultra-wideband applications," AEU - International Journal of Electronics and Communications, vol. 76, pp. 152-157, 2017.

[13] M. Çelik, "Numerical vibration analysis of a sar antenna," in 2010 IEEE Antennas and Propagation Society International Symposium, pp. 1-4, 2010. 
[14] T. Shiozumi, A. Ming, T. Kida, C. Kanamori, Y. Kobayashi, and M. Satoh, "Vibration suppression of ship-mounted antennas using a nonlinear passive vibration isolator," in 2007 IEEE International Conference on Integration Technology, pp. 568-573, 2007.

[15] H. Schippers, G. Spalluto, and G. Vos, "Radiation analysis of conformal phased array antennas on distorted structures," in Twelfth International Conference on Antennas and Propagation, 2003 (ICAP 2003). (Conf. Publ. No. 491), vol. 1, pp. 160-163 vol.1, 2003.

[16] P. Fei, Y.-C. Jiao, W. Hu, and F.-S. Zhang, "A miniaturized antipodal vivaldi antenna with improved radiation characteristics," IEEE Antennas and Wireless Propagation Letters, vol. 10, pp. 127-130, 2011.

[17] J. Bourqui, M. Okoniewski, and E. C. Fear, "Balanced antipodal vivaldi antenna with dielectric director for near-field microwave imaging," IEEE Transactions on Antennas and Propagation, vol. 58, no. 7, pp. 2318-2326, 2010.

[18] R. Kazemi, A. E. Fathy, and R. A. Sadeghzadeh, "Dielectric rod antenna array with substrate integrated waveguide planar feed network for wideband applications," IEEE Transactions on Antennas and Propagation, vol. 60, no. 3, pp. 1312-1319, 2012.

[19] L. Juan, F. Guang, Y. Lin, and F. Demin, "A modified balanced antipodal vivaldi antenna with improved radiation characteristics," Microwave and Optical Technology Letters, vol. 55, no. 6, pp. 1321-1325, 2013.

[20] C. B. Fortuny, K. Tong, K. Chetty, and P. Brittan, "High-gain triple-band reconfigurable vivaldi antenna," in 2014 IEEE-APS Topical Conference on Antennas and Propagation in Wireless Communications (APWC), pp. 199-201, 2014.

[21] N. T. Nguyen, G. Clementi, C. Migliaccio, N. Fortino, J.-Y. Dauvignac, J. Willebois, and C. Chekroun, "Wideband vivaldi antenna array with mechanical support and protection radome for land-mine detection radar," in 2015 European Radar Conference (EuRAD), pp. 537-540, 2015.

[22] M. Gatchev, S. Kamenopolsky, V. Bojanov, and P. Dankov, "Influence of the milling depth on the microstrip parameters in milled pcb-plates for microwave applications," in 14th International Conference on Microwaves, Radar and Wireless Communications. MIKON - 2002. Conference Proceedings (IEEE Cat.No.02EX562), vol. 2, pp. $476-479$ vol.2, 2002.

[23] O. Javashvili, "Uwb antennas for wall penetrating radar systems," Master's thesis, University of Gävle, Gävle, Sweden, 2009.

[24] N. Wang, M. Fang, J. Qiu, and L. Xiao, "Improved design of balanced antipodal vivaldi for mmw applications," in 2017 IEEE International Symposium on Antennas and Propagation \& USNC/URSI National Radio Science Meeting, pp. 2615-2616, 2017.

[25] A. Abbosh, H. Kan, and M. Bialkowski, "Design of compact directive ultra wideband antipodal antenna," Microwave and Optical Technology Letters, vol. 48, no. 12, pp. 2448-2450, 2006.

[26] K. H. Sayidmarie and Y. A. Fadhel, "Design aspects of uwb printed elliptical monopole antenna with impedance matching," in 2012 Loughborough Antennas \& Propagation Conference (LAPC), pp. 1-4, 2012.

[27] K. S. Yngvesson, T. Korzeniowski, Y.-S. Kim, E. L. Kollberg, and J. F. Johansson, "The tapered slot antenna-a new integrated element for millimetre-wave applications," IEEE Transactions on Microwave Theory and Techniques, vol. 37, no. 2, pp. 365-374, 1989.

[28] MultiMedia LLC, "Waves and frequency ranges," 2005. [Online]. Available: http://www.radartutorial.eu/07.waves/ Waves\%20and\%20Frequency\%20Ranges.en.html

[29] D. Fluoroproducts, “Teflon® ptfe fluoropolymer resin: properties handbook,” DuPontTM Technical Report H-37051-3, Tech. Rep., 1996. 